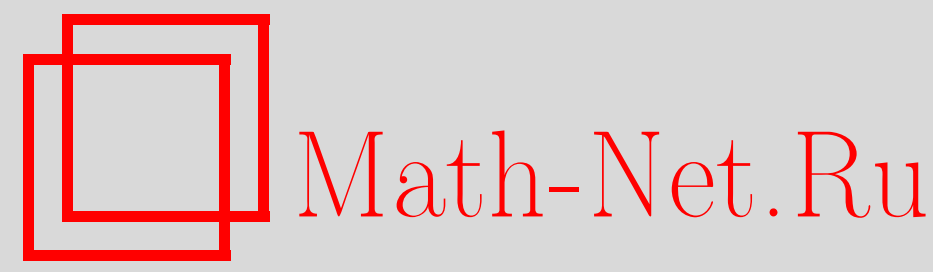

В. В. Жиков, М. Е. Рычаго, Усреднение нелинейных эллиптических уравнений второго порядка в перфорированных областях, Изв. РАН. Сер. матем., 1997, том 61, выпуск 1, 69-88

DOI: https://doi.org/10.4213/im105

Использование Общероссийского математического портала Math-Net.Ru подразумевает, что вы прочитали и согласны с пользовательским соглашением

http: //www.mathnet.ru/rus/agreement

Параметры загрузки:

IP : 3.82 .47 .9

26 апреля 2023 г., 17:28:48 
УДК 517.97

\author{
В. В. Жиков, М. Е. Рычаго
}

\title{
Усреднение нелинейных эллиптических уравнений второго порядка в перфорированных областях
}

\begin{abstract}
Классический метод усреднения эллиптических краевых задач основан на продолжении решения, заданного в перфорированной области, на всю исходную область. Этот метод требует существенных ограничений на перфорированную область (условие "сильной связности"). В работе предложен новый подход, не использующий технику продолжения. При этом "сильная связность" заменяется обычной связностью.

Библиография: 13 наименований.
\end{abstract}

\section{§1. Введение}

1. Перфорированной областью принято называть пересечение

$$
\Omega \cap Q_{\varepsilon},
$$

где $\Omega$ - фиксированная ограниченная область в $\mathbb{R}^{N}, Q_{\varepsilon}=\varepsilon Q$-гомотетическое сжатие периодического открытого множества $Q \subset \mathbb{R}^{N}$.

Усреднению линейных и нелинейных эллиптических уравнений в перфорированных областях посвящена многочисленная литература. Главные проблемы обычно связаны со структурой периодического множества $Q$. Классический метод основан на специальном продолжении решения, заданного в области (0.1), на всю исходную область $\Omega$. Этот метод требует существенных ограничений на периодическую область $Q$ (так называемое условие “сильной связности").

В настоящей работе предложен более простой подход, совсем не использующий технику продолжения. При этом все требования на периодическое открытое множество $Q$ сводятся к единственному условию - условию связности $Q$ в $\mathbb{R}^{N}$.

Рассматривается нелинейное эллиптическое уравнение вида

$$
-\operatorname{div}\left(a\left(\varepsilon^{-1} x, \nabla u^{\varepsilon}\right)\right)+b\left(\varepsilon^{-1} x, u^{\varepsilon}\right)=f
$$

в области $\Omega \cap Q_{\varepsilon}$, которое дополняется краевым условием Неймана на части $\Omega \cap \partial Q_{\varepsilon}$ границы области (0.1) и условием Дирихле - на остальной части границы. Предполагается, что $a(y, \xi), b(x, s)$ - периодические и измеримые по $x$ функции на $Q$, сильно монотонные по $\xi \in \mathbb{R}^{N}$ для п.в. $x \in Q$ и удовлетворяющие определенньм условиям роста по $\xi \in \mathbb{R}^{N}$ (см. п. 2, условия 1$\left.), 2\right)$ ).

Работа выполнена при финансовой поддержке РФФИ (проект 93-011-1720) и ISF (грант NYV000).

(C) В.В. Жиков, М.Е. РычАго 1997 
При этих предположениях мы определяем усредненное уравнение, заданное уже во всей области $\Omega$, и доказываем основные свойства сходимости решения $(0.2) \mathrm{k}$ решению усредненного уравнения.

Отметим, что в случае $Q=\mathbb{R}^{N}$ усреднение монотонных операторов изучалось многими авторами (см. [2]-[5], [12], общие свойства монотонных эллиптических операторов см. в [1], [2]).

2. Итак, пусть $Q$ - периодическая связная область в $\mathbb{R}^{N}, \square=(0,1)^{N}$ - ячейка периодичности, $\chi(x)$ - характеристическая функция $Q$,

$$
\chi(x)= \begin{cases}1, & \text { если } x \in Q, \\ 0, & \text { если } x \in F=\mathbb{R}^{N} \backslash Q .\end{cases}
$$

В перфорированной области $\Omega \cap Q_{\varepsilon}$ поставим следующую задачу:

$$
\begin{gathered}
-\operatorname{div}\left(a\left(\varepsilon^{-1} x, \nabla u^{\varepsilon}\right)\right)+b\left(\varepsilon^{-1} x, u^{\varepsilon}\right)=f \\
\left.a\left(\varepsilon^{-1} x, \nabla u^{\varepsilon}\right) \cdot n\right|_{\Omega \cap \partial Q_{\varepsilon}}=0, \\
\left.u^{\varepsilon}\right|_{\partial \Omega \cap Q_{\varepsilon}}=0
\end{gathered}
$$

где $n$ - единичная внешняя нормаль к границе. Другими словами, на части $\Omega \cap \partial Q_{\varepsilon}$ границы области $\Omega \cap Q_{\varepsilon}$ ставится условие Неймана, а на остальной части - условие Дирихле.

Свойство монотонности и свойство роста функций $a(\cdot, \xi), b(\cdot, s)$ будем характеризовать с помошью показателя $p>1$. Точнее, мы предполагаем, что функции $a(y, \xi), b(x, s), f(x)$ удовлетворяют следуюшим условиям:

1) $a(\cdot, \xi), b(\cdot, s)$ - периодические с периодом 1 и измеримые по $x$ функции на $Q$;

2) сушествует показатель $p, 1<p<\infty$, такой, что:

для п.в. $x \in Q$ и $\forall \xi_{1}, \xi_{2} \in \mathbb{R}^{N}$

i) $\left(a\left(x, \xi_{1}\right)-a\left(x, \xi_{2}\right)\right)\left(\xi_{1}-\xi_{2}\right) \geqslant \begin{cases}\alpha\left|\xi_{1}-\xi_{2}\right|^{p}, & \text { если } p \geqslant 2, \\ \alpha\left|\xi_{1}-\xi_{2}\right|^{2}\left(\left|\xi_{1}\right|+\left|\xi_{2}\right|\right)^{p-2}, & \text { если } 1<p \leqslant 2,\end{cases}$

ii) $\left|a\left(x, \xi_{1}\right)-a\left(x, \xi_{2}\right)\right| \leqslant \begin{cases}\alpha^{-1}\left(1+\left|\xi_{1}\right|+\left|\xi_{2}\right|\right)^{p-2}\left|\xi_{1}-\xi_{2}\right|, & \text { если } p \geqslant 2, \\ \alpha^{-1}\left|\xi_{1}-\xi_{2}\right|^{p-1}, & \text { если } 1<p \leqslant 2\end{cases}$

для п.в. $x \in Q$ и $\forall s_{1}, s_{2} \in \mathbb{R}^{1}$

j) $\left(b\left(x, s_{1}\right)-b\left(x, s_{2}\right)\right)\left(s_{1}-s_{2}\right) \geqslant \begin{cases}\beta\left|s_{1}-s_{2}\right|^{p}, & \text { если } p \geqslant 2, \\ \beta\left|s_{1}-s_{2}\right|^{2}\left(\left|s_{1}\right|+\left|s_{2}\right|\right)^{p-2}, & \text { если } 1<p \leqslant 2,\end{cases}$

jj) $\left|b\left(x, s_{1}\right)-b\left(x, s_{2}\right)\right| \leqslant \begin{cases}\beta^{-1}\left(1+\left|s_{1}\right|+\left|s_{2}\right|\right)^{p-2}\left|s_{1}-s_{2}\right|, & \text { если } p \geqslant 2, \\ \beta^{-1}\left|s_{1}-s_{2}\right|^{p-1}, & \text { если } 1<p \leqslant 2,\end{cases}$ где $\alpha, \beta$ - положительные константы;

3) $a(x, 0)=b(x, 0)=0, \quad f(x) \in L^{p^{\prime}}(\Omega), \quad p^{\prime}=\frac{p}{p-1}$.

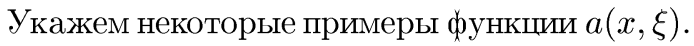

ПРИМЕР 1. Пусть

$$
a(x, \xi)=|\xi|^{p-2} \xi, \quad b(x, s)=|s|^{p-2} s .
$$

Тогда (1.1) есть уравнение Эйлера соответствующей вариационной задачи. 
ПримеР 2. Пусть $a(x, \xi)=a(x) \xi$, где матрица $a(x)=\left(a_{i j}(x)\right)$, не обязательно симметрическая, такова, что:

a) $a_{i j} \in L^{\infty}\left(\mathbb{R}^{N}\right) \forall i, j=1, \ldots, N$;

б) $\exists \alpha>0$ такое, что

$$
a(x) \xi \cdot \xi=\sum_{i, j=1}^{N} a_{i j}(x) \xi_{j} \xi_{i} \geqslant \alpha|\xi|^{2}
$$

для п.в. $x \in \mathbb{R}^{N}$ и $\forall \xi \in \mathbb{R}^{1}$.

Непосредственно проверяется, что определенная таким образом функция $a(x, \xi)$ удовлетворяет условиям 1)-3) с $p=2$.

3. Чтобы точно сформулировать задачу (1.1), введем пространство $V_{\varepsilon}$, равное замыканию множества $C_{0}^{\infty}(\Omega)$ по норме

$$
\left(\int_{\Omega \cap Q_{\varepsilon}}\left(|\nabla u|^{p}+|u|^{p}\right) d x\right)^{1 / p} .
$$

ОПРЕДЕЛЕНИЕ. Функция $u^{\varepsilon} \in V_{\varepsilon}$ называется решением задачи (1.1), если

$$
\int_{\Omega \cap Q_{\varepsilon}} a\left(\varepsilon^{-1} x, \nabla u^{\varepsilon}\right) \cdot \nabla \varphi d x+\int_{\Omega \cap Q_{\varepsilon}} b\left(\varepsilon^{-1} x, u^{\varepsilon}\right) \varphi d x=\int_{\Omega \cap Q_{\varepsilon}} f \varphi d x \quad \forall \varphi \in V_{\varepsilon} .
$$

Из теории монотонных эллиптических операторов (см. [1], [2]) следует, что задача (1.1) имеет единственное решение $u^{\varepsilon} \in V_{\varepsilon}$. Возьмем в (1.3) $\varphi=u^{\varepsilon}$. Тогда, применяя свойства ii) и јj) для $a$ и $b$ соответственно, получим такую оценку:

$$
\int_{\Omega \cap Q_{\varepsilon}}\left|\nabla u^{\varepsilon}\right|^{p} d x+\int_{\Omega \cap Q_{\varepsilon}}\left|u^{\varepsilon}\right|^{p} d x \leqslant C \int_{\Omega \cap Q_{\varepsilon}}|f|^{p^{\prime}} d x
$$

где константа $C$ не зависит от $\varepsilon$.

Решение $u^{\varepsilon}$ задачи (1.1) фактически определено только в перфорированной области $\Omega \cap Q_{\varepsilon}$, и в этом состоит главная трудность при изучении его поведения при $\varepsilon \rightarrow 0$. Представляется естественным предварительно продолжить это решение $u^{\varepsilon}$ на всю область $\Omega$ до функции $\tilde{u}^{\varepsilon} \in W^{1, p}(\Omega)$ и изучать последовательность продолжений $\tilde{u}^{\varepsilon}(x)$. На идее продолжения базируются многочисленные работы по усреднению в перфорированных областях (см. [8]- [11]). Этот метод требует операторов продолжения со специальными оценками типа

$$
\int_{\Omega}\left|\nabla \tilde{u}^{\varepsilon}\right|^{p} d x \leqslant c_{0} \int_{\Omega}\left|\nabla u^{\varepsilon}\right|^{p} d x
$$

где константа $c_{0}$ не зависит от $\varepsilon$. Существование таких операторов возможно только при определенных ограничениях на периодическую область $Q$ (так называемое условие "сильной связности"). Наиболее общая формулировка такова: подходящие операторы продолжения сушествуют, если $Q$ связна и удовлетворяет условию Липшица (см. [11]). Для нелипшицевых областей такие операторы могут не 
сушествовать, например, если $\mathbb{R}^{3} \backslash Q$ - плотная кубическая упаковка шаров в $\mathbb{R}^{3}$. Заметим, что в этом примере $Q$ связна.

Настоящая работа опирается на более простой подход (см. [6], [7]), совсем не используюший технику продолжения в соболевских пространствах. При этом на периодическую область $Q$ накладьвается только условие связности в $\mathbb{R}^{N}$. Интересно, что возможно и некоторое ослабление условия связности (так называемое условие $p$-связности). Этот вопрос обсуждается в работе [6].

4. Введем вспомогательную периодическую задачу. По существу она должна иметь вид

$$
\begin{gathered}
u(y)-\text { периодическая функция, } \\
\operatorname{div}(a(y, \xi+\nabla u(y)))=0 \quad \text { в } Q, \\
\left.a(y, \xi+\nabla u(y)) \cdot n\right|_{\partial Q}=0,
\end{gathered}
$$

где $\xi$ - фиксированный вектор из $\mathbb{R}^{N}$ (так что решение зависит от $\xi, u=u(y, \xi)$ ).

Однако при наших условиях на $Q$ мы можем гарантировать не свойства потенциала $u(x)$, а лишь свойства градиента $\nabla u(x)$. В связи с этим вспомогательную периодическую задачу переформулируем таким образом.

Пусть $C_{\mathrm{per}}^{\infty}(\square)$ - множество всех гладких периодических функций, $\mathfrak{V}_{\mathrm{pot}}^{p}(Q)-$ замыкание множества $\left\{\nabla \varphi: \varphi \in C_{\text {per }}^{\infty}(\square)\right\}$ по норме

$$
\left(\int_{Q}|\nabla \varphi|^{p} d x\right)^{1 / p}
$$

Элементы пространства $\mathscr{V}_{\text {pot }}^{p}(Q)$ состоят из потенциальных периодических векторов, заданных в области $Q$. Теперь задача (1.6) имеет вид

$$
v \in \mathscr{V}_{\text {pot }}^{p}(Q), \quad \operatorname{div}(\chi(y) a(y, \xi+v))=0
$$

Это означает, что для решения задачи (1.7) должно выполняться тождество

$$
\int_{\square \cap Q} a(y, \xi+v) \cdot \psi d x=0 \quad \forall \psi \in \mathscr{V}_{\mathrm{pot}}^{p}(Q) .
$$

Согласно теории монотонных операторов вспомогательная периодическая задача (1.7) имеет единственное решение.

С помощью задачи (1.7) определим усредненный оператор

$$
a_{0}(\xi)=\int_{\square \cap Q} a(y, \xi+v(y)) d y
$$

Используя определение $a_{0}$ и свойства 2$)$ и 3$)$ для $a(y, \xi)$, можно непосредственно показать, что усредненный оператор также монотонный, коэрцитивный и удовлетворяет условиям типа іi). (Доказательство этих фактов приведем в $\S 4$.) 
Определим также оператор

$$
b_{0}(s)=\int_{\square \cap Q} b(y, s) d y
$$

Очевидно, что оператор $b_{0}(s)$ тоже удовлетворяет условиям сильной монотонности. Кроме того, в силу условия jj) для $b$ справедливо следуюшее соотношение

$$
\left|b_{0}\left(s_{1}\right)-b_{0}\left(s_{2}\right)\right| \leqslant \begin{cases}\beta_{0}\left(1+\left|s_{1}\right|+\left|s_{2}\right|\right)^{p-2}\left|s_{1}-s_{2}\right|, & \text { если } p \geqslant 2, \\ \beta_{0}\left|s_{1}-s_{2}\right|^{p-1}, & \text { если } 1<p \leqslant 2 .\end{cases}
$$

Таким образом, мы можем ввести усредненную задачу

$$
\begin{gathered}
-\operatorname{div}\left(a_{0}\left(\nabla u^{0}\right)\right)+b\left(u^{0}\right)=\theta f, \\
u^{0} \in W_{0}^{1, p}(\Omega),
\end{gathered}
$$

где $\theta=\int_{\square} \chi(x) d x$-плотность множества $Q$. Ввиду указанных вьше свойств $a_{0}$ и $b_{0}$ усредненная задача (1.12) имеет единственное решение.

Пусть

$$
\chi_{\varepsilon}(x)=\chi\left(\varepsilon^{-1} x\right)= \begin{cases}1, & \text { если } x \in Q_{\varepsilon}, \\ 0, & \text { если } x \in \mathbb{R}^{N} \backslash Q_{\varepsilon} .\end{cases}
$$

Сформулируем основной результат работы.

ТЕОРема 1.1. Предположим, что периодическое открытое множество $Q$ связно в $\mathbb{R}^{N}$ и функции $a(y, \xi), b(x, s)$ удовлетворяют условиям 1)-3).

Тогда имеет место слабая сходимость потоков:

$$
\begin{aligned}
& \chi_{\varepsilon}(x) a\left(\varepsilon^{-1} x, \nabla u^{\varepsilon}\right) \rightarrow a_{0}\left(\nabla u^{0}\right) \quad \text { слабо в } \quad L^{p^{\prime}}(\Omega), \\
& \chi_{\varepsilon}(x) b\left(\varepsilon^{-1} x, u^{\varepsilon}\right) \rightarrow b_{0}\left(u^{0}\right) \quad \text { слабо в } \quad L^{p^{\prime}}(\Omega),
\end{aligned}
$$

а также справедлива сильная сходимость решений в следующем смысле:

$$
\lim _{\varepsilon \rightarrow 0} \int_{\Omega \cap Q_{\varepsilon}}\left|u^{\varepsilon}-u^{0}\right|^{p} d x=0
$$

где $u^{\varepsilon}$ - решение задачи (1.1), а и $u^{0}$ - решение усредненной задачи (1.12).

Ниже мы покажем, что условия теоремы 1.1 могут быть ослаблены. В частности, можно рассматривать функцию

$$
b(x, u)=\lambda u(x), \quad \lambda>0 .
$$

Этот случай важен для параболических уравнений. 


\section{§2. Вспомогательные результаты}

В этом параграфе мы изложим некоторые факты, которые нам понадобятся в дальнейшем.

Напомним хорошо известное свойство среднего значения периодических функций.

Пусть $q(x)$ - периодическая функция, $q \in L_{\mathrm{per}}^{\alpha}(\square), \alpha \geqslant 1$. Тогда при $\varepsilon \rightarrow 0$

$$
q\left(\varepsilon^{-1} x\right) \rightarrow\langle q\rangle=\int_{\square} q(x) d x \quad \text { слабо в } L^{\alpha}(\square) .
$$

Далее, для доказательства основной теоремы мы используем некоторые обобшения леммы о компенсированной компактности. Дадим сначала формулировку этой леммы.

ЛЕмма 2.1 (см. [9, с. 12]). Пусть $p^{\varepsilon}, v^{\varepsilon}-$ векторь из $L^{p}(\Omega)$,

$$
p^{\varepsilon} \rightarrow p^{0}, \quad v^{\varepsilon} \rightarrow v^{0} \quad \text { слабо в } \quad L^{p}(\Omega) .
$$

Если дополнительно $\operatorname{rot} v^{\varepsilon}=0 u \operatorname{div} p^{\varepsilon}=f^{\varepsilon}$, әде $f^{\varepsilon} \rightarrow f$ слабо в $L^{p^{\prime}}(\Omega)$, то $n p u \varepsilon \rightarrow 0$

$$
p^{\varepsilon} \cdot v^{\varepsilon} \stackrel{*}{\rightarrow} p^{0} \cdot v^{0} \quad \text { в } \quad L^{1}(\Omega)
$$

m.e.

$$
\int_{\Omega} \varphi p^{\varepsilon} \cdot v^{\varepsilon} d x=\int_{\Omega} \varphi p^{0} \cdot v^{0} d x \quad \forall \varphi \in C_{0}^{\infty}(\Omega) .
$$

Приведем некоторое уточнение леммы 2.1. Для этого напомним известный критерий слабой компактности в $L^{1}(\Omega)$ (см. [13, с. 238]).

Следующие утверждения равносильнь:

а) последовательность $w^{\varepsilon} \in L^{1}$ слабо компактна в $L^{1}(\Omega)$;

b) последовательность $w^{\varepsilon}$ равностепенно интегрируема, т.е. для любого натурального $n$ найдется $\delta=\delta(n)$ такое, что

$$
\int_{A}\left|w^{\varepsilon}\right| d x \leqslant \frac{1}{n}
$$

для $\forall \varepsilon>0$ и всякого измеримого множества $A$ с $|A| \leqslant \delta$;

c) $\lim _{N \rightarrow+\infty} \sup _{\varepsilon} \int_{\left|w^{\varepsilon}\right| \geqslant N}\left|w^{\varepsilon}\right| d x=0$.

Имеет место следующая лемма.

Лемма 2.2. Пусть $p(x) \in\left(L^{p^{\prime}}(\Omega)\right)^{N}$,

$$
p^{\varepsilon} \rightarrow p^{0} \quad \text { cлабо в } \quad L^{p^{\prime}}(\Omega)
$$

причем $\operatorname{div} p^{\varepsilon}=f^{\varepsilon}$, где $f^{\varepsilon} \rightarrow f$ слабо в $L^{p^{\prime}}(\Omega)$.

Тогда если $v(x) \in L_{\mathrm{per}}^{p}(\square)$ - потенииальный периодический вектор, $v_{\varepsilon}=$ $v\left(\varepsilon^{-1} x\right)$, mо при $\varepsilon \rightarrow 0$

$$
p^{\varepsilon} \cdot v_{\varepsilon} \rightarrow p^{0} \cdot\langle v\rangle \quad \text { слабо в } \quad L^{1}(\Omega) .
$$


ДокАЗАТЕЛЬСТВо. Так как $p^{\varepsilon} \cdot v_{\varepsilon} \stackrel{*}{\rightarrow} p^{0} \cdot\langle v\rangle$ по лемме 2.1 , то достаточно проверить, что последовательность $p^{\varepsilon} \cdot v_{\varepsilon}$ слабо компактна в $L^{1}(\Omega)$.

Заметим, что последовательность $\left|v_{\varepsilon}\right|^{p}$ не только ограничена в $L^{1}(\Omega)$, но и слабо компактна в $L^{1}(\Omega)$, так как по свойству среднего значения она слабо сходится в $L^{1}(\Omega)$ к $\left\langle|v|^{p}\right\rangle$. Поэтому в силу критерия слабой компактности семейство $\left|v_{\varepsilon}\right|^{p}$ равностепенно интегрируемо. Но тогда и семейство $p^{\varepsilon} \cdot v_{\varepsilon}$ равностепенно интегрируемо, так как

$$
\int_{A}\left|p^{\varepsilon} \cdot v_{\varepsilon}\right| d x \leqslant\left(\int_{A \cap Q_{\varepsilon}}\left|p^{\varepsilon}\right|^{p^{\prime}} d x\right)^{1 / p^{\prime}} \cdot\left(\int_{A}\left|v_{\varepsilon}\right|^{p} d x\right)^{1 / p} \leqslant C \delta,
$$

если только $|A|$ достаточно мало.

Итак, последовательность $p^{\varepsilon} \cdot v_{\varepsilon}$ слабо компактна в $L^{1}(\Omega)$, и лемма доказана.

Следуюший факт не столь тривиален. Пусть дана последовательность $u^{\varepsilon} \in V_{\varepsilon}$ (см. определение (1.2) пространства $V_{\varepsilon}$ ) такая, что

$$
\varlimsup_{\varepsilon \rightarrow 0} \int_{\Omega \cap Q_{\varepsilon}}\left|u^{\varepsilon}\right|^{p} d x<+\infty, \quad \varlimsup_{\varepsilon \rightarrow 0} \int_{\Omega \cap Q_{\varepsilon}}\left|\nabla u^{\varepsilon}\right|^{p} d x<+\infty .
$$

Иначе говоря, последовательности

$$
\chi_{\varepsilon} u^{\varepsilon}, \quad \chi_{\varepsilon} \nabla u^{\varepsilon} \quad \text { ограничены в } L^{p}(\Omega) \text {. }
$$

Без потери обшности считаем, что

$$
\chi_{\varepsilon} u^{\varepsilon} \rightarrow \theta u^{0} \quad \text { слабо в } L^{p}(\Omega) .
$$

Справедлива такая лемма.

ЛЕмма 2.3. Пусть последовательность $u^{\varepsilon} \in V_{\varepsilon}$ удовлетворяет условия.м (2.1), (2.3). Тогда $u^{0} \in W_{0}^{1, p}(\Omega) u$

$$
\int_{\Omega}\left|\nabla u^{0}\right|^{p} d x \leqslant c_{0} \varlimsup_{\varepsilon \rightarrow 0} \int_{\Omega \cap Q_{\varepsilon}}\left|\nabla u^{\varepsilon}\right|^{p} d x,
$$

где константа с с зависит только от области $Q$.

Кроме того, если

$$
z \in\left(L_{\text {per }}^{p^{\prime}}(\square)\right)^{N}, \quad \operatorname{div} z=0,\left.\quad z\right|_{\mathbb{R}^{N} \backslash Q}=0,
$$

mo $n p u \varepsilon \rightarrow 0$

$$
\nabla u^{\varepsilon} \cdot z\left(\varepsilon^{-1} x\right) \rightarrow \nabla u^{0} \cdot\langle z\rangle \quad \text { слабо в } \quad L^{1}(\Omega) .
$$

ДокАЗАТЕЛЬСТво этой леммы дано в работе [6, с. 398]. 


\section{§3. Доказательство основной теоремы}

1. Пусть $u^{\varepsilon}$ - решение задачи (1.1). Оценка (1.4) означает, что для этого решения $u^{\varepsilon}$ выполнены условия (2.1). Без потери обшности считаем, что имеет место слабая сходимость (2.3).

Далее положим

$$
p_{\varepsilon}(x)=\chi_{\varepsilon} a\left(\varepsilon^{-1} x, \nabla u^{\varepsilon}\right), \quad h_{\varepsilon}(x)=\chi_{\varepsilon} b\left(\varepsilon^{-1} x, u^{\varepsilon}\right) .
$$

Тогда имеем такое уравнение:

$$
-\operatorname{div} p_{\varepsilon}+h_{\varepsilon}=\chi_{\varepsilon} f \quad \text { в } \Omega .
$$

Из условий ii $), \mathrm{jj})$ для $a(y, \xi)$ и $b(x, s)$ следует, что

$$
p_{\varepsilon}(x), \quad h_{\varepsilon}(x) \text { ограничены в } L^{p^{\prime}}(\Omega) .
$$

Без ограничения общности считаем, что

$$
p_{\varepsilon} \rightarrow p_{0}, \quad h_{\varepsilon} \rightarrow h_{0} \quad \text { слабо в } L^{p^{\prime}}(\Omega) .
$$

Тогда, переходя к пределу в (3.2), получим

$$
-\operatorname{div} p_{0}+h_{0}=\theta f
$$

Очевидно, требуется проверить равенства

$$
p_{0}=a_{0}\left(\nabla u^{0}\right), \quad h_{0}=b_{0}\left(u^{0}\right) .
$$

Тогда в силу единственности решения усредненной задачи (1.12) соотношения (1.13) и (1.14) будут доказаны. После этого нам останется доказать сильную сходимость решений в смысле (1.15).

2. Пусть $v(y, \xi)$ - решение вспомогательной периодической задачи (1.7). Тогда по определению пространства $\mathscr{V}_{\text {pot }}^{p}(Q) \forall \delta>0$ найдется вектор $N^{\delta}(y)=N^{\delta}(y, \xi) \in$ $C_{\text {per }}^{\infty}(\square)$ такой, что

$$
\lim _{\delta \rightarrow 0} \int_{\square \cap Q}\left|v-\nabla N^{\delta}\right|^{p} d x=0 .
$$

Очевидно, что при этом

$$
\lim _{\delta \rightarrow 0} \int_{\square \cap Q} a\left(y, \xi+\nabla N^{\delta}\right) d x=a_{0}(\xi) .
$$

Кроме того, ввиду (1.9) и (3.8) справедливо следуюшее соотношение:

$$
\lim _{\delta \rightarrow 0}\left\|\chi(y)\left(a(y, \xi+v)-a\left(y, \xi+\nabla N^{\delta}\right)\right)\right\|_{L^{p^{\prime}(\square)}}=0 .
$$


Теперь введем пробную функцию

$$
w^{\varepsilon}(x)=\xi \cdot x+\varepsilon N^{\delta}(y), \quad y=\varepsilon^{-1} x,
$$

и заметим, что $\nabla w^{\varepsilon}=\xi+\nabla N^{\delta}, w^{\varepsilon} \rightarrow \xi \cdot x$ в $W_{\text {loc }}^{1, p}\left(\mathbb{R}^{N}\right)$.

Через $K$ обозначим произвольную подобласть области $\Omega$. По монотонности имеем неравенство

$$
\int_{K} \chi_{\varepsilon}\left(a\left(\varepsilon^{-1} x, \nabla u^{\varepsilon}\right)-a\left(\varepsilon^{-1} x, \nabla w^{\varepsilon}\right)\right) \cdot\left(\nabla u^{\varepsilon}-\nabla w^{\varepsilon}\right) d x \geqslant 0,
$$

левая часть которого содержит четыре слагаемых, и в каждом из них требуется перейти к пределу сначала при $\varepsilon \rightarrow 0$, а затем при $\delta \rightarrow 0$.

Для первого слагаемого имеем

$$
\begin{aligned}
\lim _{\varepsilon \rightarrow 0} \mathscr{I}_{\varepsilon}^{1} & =\lim _{\varepsilon \rightarrow 0} \int_{K} \chi_{\varepsilon} a\left(\varepsilon^{-1} x, \nabla w^{\varepsilon}\right) \cdot \nabla w^{\varepsilon} d x \\
& =\int_{K}\left\langle\chi(y) a\left(y, \xi+\nabla N^{\delta}\right) \cdot\left(\xi+\nabla N^{\delta}\right)\right\rangle d x
\end{aligned}
$$

по свойству среднего значения. Отсюда в силу (3.7), (3.8)

$$
\lim _{\delta \rightarrow 0} \lim _{\varepsilon \rightarrow 0} \mathscr{I}_{\varepsilon}^{1}=\int_{K} a_{0}(\xi) \cdot \xi d x
$$

Рассмотрим второе слагаемое из (3.10):

$$
\begin{aligned}
\lim _{\varepsilon \rightarrow 0} \mathscr{I}_{\varepsilon}^{2} & =\lim _{\varepsilon \rightarrow 0} \int_{K} \chi_{\varepsilon} a\left(\varepsilon^{-1} x, \nabla w^{\varepsilon}\right) \cdot \nabla u^{\varepsilon} d x \\
& =\int_{K}\left\langle\chi(y) a\left(y, \xi+\nabla N^{\delta}\right)\right\rangle \cdot \nabla u^{0} d x .
\end{aligned}
$$

Здесь мы воспользовались леммой 2.3. Далее, согласно (3.8)

$$
\lim _{\delta \rightarrow 0} \lim _{\varepsilon \rightarrow 0} \mathscr{I}_{\varepsilon}^{2}=\int_{K} a_{0}(\xi) \cdot \nabla u^{0} d x
$$

Для третьего слагаемого имеем

$$
\lim _{\varepsilon \rightarrow 0} \mathscr{I}_{\varepsilon}^{3}=\lim _{\varepsilon \rightarrow 0} \int_{K} \chi_{\varepsilon} a\left(\varepsilon^{-1} x, \nabla u^{\varepsilon}\right) \cdot \nabla w^{\varepsilon} d x=\int_{K} p_{0} \cdot \xi d x
$$

в силу леммы 2.2 .

Оставшееся слагаемое

$$
\lim _{\varepsilon \rightarrow 0} \int_{K} \chi_{\varepsilon} a\left(\varepsilon^{-1} x, \nabla u^{\varepsilon}\right) \cdot \nabla u^{\varepsilon} d x
$$

мы пока не вычисляем (заметим только, что оно не зависит от выбора пробной функции $w^{\varepsilon}(x)$ ). 
Таким образом, неравенство (3.10) принимает вид

$$
\lim _{\varepsilon \rightarrow 0} \int_{K} p_{\varepsilon} \cdot \nabla u^{\varepsilon} d x+\int_{K} a_{0}(\xi) \cdot \xi d x-\int_{K} a_{0}(\xi) \cdot \nabla u^{0} d x-\int_{K} p_{0} \cdot \xi d x \geqslant 0
$$

Теперь мы распространим его на кусочно аффинные функции $\omega(x) \in W_{0}^{1, p}(\Omega)$.

Пусть $\Omega_{j} \subset \Omega$ есть участки аффинности $\omega(x),\left.\nabla \omega\right|_{\Omega_{j}}=\xi^{j}$. Возьмем в $(3.11)$ $K=\Omega_{j}$ и $\xi=\xi^{j} ;$ получим

$$
\begin{aligned}
\lim _{\varepsilon \rightarrow 0} \int_{\Omega} p_{\varepsilon} \cdot \nabla u^{\varepsilon} d x & +\int_{\Omega} a_{0}(\nabla \omega) \cdot \nabla \omega d x-\int_{\Omega} a_{0}(\nabla \omega) \cdot \nabla u^{0} d x \\
& -\int_{\Omega} p_{0} \cdot \nabla \omega d x \geqslant 0 .
\end{aligned}
$$

Далее проведем аналогичные рассуждения для слагаемого $h_{\varepsilon}=\chi_{\varepsilon} b\left(\varepsilon^{-1} x, u^{\varepsilon}\right)$. Имеем по монотонности

$$
\int_{K} \chi_{\varepsilon}\left(b\left(\varepsilon^{-1} x, u^{\varepsilon}\right)-b\left(\varepsilon^{-1} x, \eta\right)\right) \cdot\left(u^{\varepsilon}-\eta\right) d x \geqslant 0
$$

Рассмотрим первое слагаемое из (3.13). По свойству среднего значения

$$
\lim _{\varepsilon \rightarrow 0} \int_{K} \chi_{\varepsilon} b\left(\varepsilon^{-1} x, \eta\right) \eta d x=\int_{K}\langle\chi(y) b(y, \eta) \eta\rangle d x=\int_{K} b_{0}(\eta) \eta d x .
$$

Для вычисления второго слагаемого, именно

$$
\lim _{\varepsilon \rightarrow 0} \int_{K} \chi_{\varepsilon} b\left(\varepsilon^{-1} x, \eta\right) u^{\varepsilon} d x
$$

нам потребуется следующая лемма.

ЛЕмма 3.1 (см. [6, с. 400]). . Пусть

$$
g(x) \in L_{\text {per }}^{p^{\prime}}(\square),\left.\quad g\right|_{\mathbb{R}^{N} \backslash Q}=0, \quad\langle g\rangle=0 .
$$

$\operatorname{Ecлu~} w^{\varepsilon} \in C^{\infty}(\bar{\Omega}) u$

$$
\lim _{\varepsilon \rightarrow 0} \int_{\Omega} \chi_{\varepsilon}\left(\left|w^{\varepsilon}\right|^{p}+\left|\nabla w^{\varepsilon}\right|^{p}\right) d x<+\infty
$$

mo

$$
g\left(\varepsilon^{-1} x\right) w^{\varepsilon}(x) \rightarrow 0 \quad \text { слабо в } \quad L^{1}(\Omega) .
$$

Итак, по лемме 3.1 имеем

$$
\begin{aligned}
\lim _{\varepsilon \rightarrow 0} \int_{K} \chi_{\varepsilon} b\left(\varepsilon^{-1} x, \eta\right) u^{\varepsilon} d x= & \lim _{\varepsilon \rightarrow 0} \int_{K} \chi_{\varepsilon}\left(b\left(\varepsilon^{-1} x, \eta\right)-\theta^{-1} b_{0}(\eta)\right) u^{\varepsilon} d x \\
& +\lim _{\varepsilon \rightarrow 0} \int_{K} \chi_{\varepsilon} \theta^{-1} b_{0}(\eta) u^{\varepsilon} d x=\int_{K} b_{0}(\eta) u^{0} d x .
\end{aligned}
$$


Кроме того, согласно (3.4)

$$
\lim _{\varepsilon \rightarrow 0} \int_{K} h_{\varepsilon} \eta d x=\int_{K} h_{0} \eta d x .
$$

Таким образом,

$$
\lim _{\varepsilon \rightarrow 0} \int_{K} h_{\varepsilon} u^{\varepsilon} d x+\int_{K} b_{0}(\eta) \eta d x-\int_{K} b_{0}(\eta) u^{0} d x-\int_{K} h_{0} \eta d x \geqslant 0 .
$$

Рассмотрим кусочно постоянную функцию $\lambda(x)$ и возьмем в (3.14) $K=\Omega_{j}$, $\eta=\eta_{j}$. Здесь $\Omega_{j} \subset \Omega:\left.\lambda(x)\right|_{\Omega_{j}}=\eta_{j}$. Тогда получим следующее неравенство:

$$
\begin{aligned}
\lim _{\varepsilon \rightarrow 0} \int_{\Omega} h_{\varepsilon}(x) u^{\varepsilon} d x & +\int_{\Omega} b_{0}(\lambda(x)) \lambda(x) d x \\
& -\int_{\Omega} b_{0}(\lambda(x)) u^{0}(x) d x-\int_{\Omega} h_{0}(x) \lambda(x) d x \geqslant 0 .
\end{aligned}
$$

После этого, складьвая неравенства (3.12) и (3.15), имеем

$$
\begin{aligned}
\lim _{\varepsilon \rightarrow 0} \int_{\Omega} p_{\varepsilon} \cdot \nabla u^{\varepsilon} d x & +\lim _{\varepsilon \rightarrow 0} \int_{\Omega} h_{\varepsilon} u^{\varepsilon} d x+\int_{\Omega} a_{0}(\nabla \omega) \cdot \nabla \omega d x \\
& -\int_{\Omega} a_{0}(\nabla \omega) \cdot \nabla u^{0} d x-\int_{\Omega} p_{0} \cdot \nabla \omega d x \\
& +\int_{\Omega} b_{0}(\lambda) \lambda d x-\int_{\Omega} b_{0}(\lambda) u^{0} d x-\int_{\Omega} h_{0} \lambda d x \geqslant 0
\end{aligned}
$$

По основному тождеству (1.3)

$$
\lim _{\varepsilon \rightarrow 0} \int_{\Omega} p_{\varepsilon} \cdot \nabla u^{\varepsilon} d x+\lim _{\varepsilon \rightarrow 0} \int_{\Omega} h_{\varepsilon} u^{\varepsilon} d x=\theta \int_{\Omega} f u^{0} d x
$$

Сравнивая это равенство с (3.5), получим

$$
\lim _{\varepsilon \rightarrow 0} \int_{\Omega} p_{\varepsilon} \cdot \nabla u^{\varepsilon} d x+\lim _{\varepsilon \rightarrow 0} \int_{\Omega} h_{\varepsilon} u^{\varepsilon} d x=\int_{\Omega} p_{0} \cdot \nabla u^{0} d x+\int_{\Omega} h_{0} u^{0} d x .
$$

Поэтому неравенство (3.16) окончательно примет вид

$$
\int_{\Omega}\left(p_{0}-a_{0}(\nabla \omega)\right) \cdot\left(\nabla u^{0}-\nabla \omega\right) d x+\int_{\Omega}\left(h_{0}-b_{0}(\lambda)\right)\left(u^{0}-\lambda\right) d x \geqslant 0 .
$$

Из оценок типа ii) для $a_{0}($ см. $\S 4)$ и (1.11) для $b_{0}$ заключаем, что в последнем неравенстве в качестве $\omega(x)$ и $\lambda(x)$ можно взять произвольные функции из $W_{0}^{1, p}(\Omega)$ и $L^{p}(\Omega)$ соответственно. 
Остается воспользоваться известными рассуждениями Минти (см., например, $\left[1\right.$, c. 184]). Именно, возьмем в $(3.19)$ вместо $\omega(x)$ и $\lambda(x)$ функции $u^{0}+t \cdot k$ и $u^{0}+t \cdot l$ соответственно, где $k(x) \in W_{0}^{1, p}(\Omega), l(x) \in L^{p}(\Omega), t \in \mathbb{R}^{1}$. В результате получим

$$
-t \int_{\Omega}\left(p_{0}-a_{0}\left(\nabla u^{0}+t \cdot \nabla k\right)\right) \cdot \nabla k d x-\int_{\Omega}\left(h_{0}-b_{0}\left(u^{0}+t l\right)\right) \cdot l(x) d x \geqslant 0 .
$$

Переходя к пределу при $t \rightarrow 0$ и сокращая на $t$, получаем

$$
\int_{\Omega}\left(p_{0}-a_{0}\left(\nabla u^{0}\right)\right) \cdot \nabla k(x) d x+\int_{\Omega}\left(h_{0}-b_{0}\left(u^{0}\right)\right) l(x) d x=0 .
$$

Отсюда ввиду произвольности функций $k(x), l(x)$ следует, что

$$
\operatorname{div} p_{0}=\operatorname{div} a_{0}\left(\nabla u^{0}\right), \quad h_{0}=b_{0}\left(u^{0}\right)
$$

Таким образом, доказано, что предельная функция $u^{0}$ есть решение усредненного уравнения (1.12). Кроме того, доказана слабая сходимость

$$
\chi_{\varepsilon}(x) b\left(\varepsilon^{-1} x, u^{\varepsilon}\right) \rightarrow b_{0}\left(u^{0}\right) \quad \text { слабо в } L^{p^{\prime}}(\Omega) .
$$

3. Установим теперь сильную сходимость решений в смысле (1.15). Полагая $\omega(x)=u^{0}(x)$ в (3.12) и $\lambda(x)=u^{0}(x)$ в (3.15), получим следуюшие неравенства:

$$
\begin{aligned}
\lim _{\varepsilon \rightarrow 0} \int_{\Omega} p_{\varepsilon} \cdot \nabla u^{\varepsilon} d x \geqslant \int_{\Omega} p_{0} \cdot \nabla u^{0} d x, \\
\lim _{\varepsilon \rightarrow 0} \int_{\Omega} h_{\varepsilon} u^{\varepsilon} d x \geqslant \int_{\Omega} h_{0} u^{0} d x
\end{aligned}
$$

что в сравнении с (3.18) означает точные равенства

$$
\begin{aligned}
\lim _{\varepsilon \rightarrow 0} \int_{\Omega} p_{\varepsilon} \cdot \nabla u^{\varepsilon} d x & =\int_{\Omega} p_{0} \cdot \nabla u^{0} d x, \\
\lim _{\varepsilon \rightarrow 0} \int_{\Omega} \chi_{\varepsilon} b\left(\varepsilon^{-1} x, u^{\varepsilon}\right) u^{\varepsilon} d x & =\lim _{\varepsilon \rightarrow 0} \int_{\Omega} \chi_{\varepsilon} b\left(\varepsilon^{-1} x, u^{\varepsilon}\right) u^{0} d x .
\end{aligned}
$$

Последнее соотношение можно переписать в виде

$$
\begin{aligned}
& \lim _{\varepsilon \rightarrow 0} \int_{\Omega} \chi_{\varepsilon}\left(b\left(\varepsilon^{-1} x, u^{\varepsilon}\right)-b\left(\varepsilon^{-1} x, u^{0}\right)\right)\left(u^{\varepsilon}-u^{0}\right) d x \\
& \quad+\lim _{\varepsilon \rightarrow 0} \int_{\Omega} \chi_{\varepsilon} b\left(\varepsilon^{-1} x, u^{0}\right)\left(u^{\varepsilon}-u^{0}\right) d x=0 .
\end{aligned}
$$

Заметим, что до сих пор мы использовали лишь монотонность $a(y, \xi)$ и $b(x, s)$. Теперь воспользуемся сильной монотонностью функции $b(x, s)$. Покажем, что

$$
\lim _{\varepsilon \rightarrow 0} \int_{\Omega} \chi_{\varepsilon} b\left(\varepsilon^{-1} x, u^{0}\right)\left(u^{\varepsilon}-u^{0}\right) d x=0
$$


Для этого левую часть (3.23) запишем так:

$$
\begin{aligned}
\int_{\Omega} \chi_{\varepsilon} & \left(b\left(\varepsilon^{-1} x, u^{0}\right)-\theta^{-1} b_{0}\left(u^{0}\right)\right)\left(u^{\varepsilon}-u^{0}\right) d x \\
& +\int_{\Omega} \chi_{\varepsilon} \theta^{-1} b_{0}\left(u^{0}\right)\left(u^{\varepsilon}-u^{0}\right) d x=I_{1}^{\varepsilon}+I_{2}^{\varepsilon} .
\end{aligned}
$$

Очевидно, что $\lim _{\varepsilon \rightarrow 0} I_{2}^{\varepsilon}=0$ в силу $(3.2)$. Для изучения слагаемого $I_{1}^{\varepsilon}$ обозначим

$$
g\left(\varepsilon^{-1} x, u^{0}\right)=\chi_{\varepsilon}\left(b\left(\varepsilon^{-1} x, u^{0}\right)-\theta^{-1} b_{0}\left(u^{0}\right)\right)
$$

и возьмем кусочно постоянную функцию $u^{\delta}$ такую, что

$$
\int_{\Omega}\left|u^{0}-u^{\delta}\right|^{p} d x \leqslant \delta \quad \forall \delta>0 .
$$

Тогда в силу условий $\mathrm{jj})$ для $b(x, s)$

$$
\int_{\Omega}\left|g\left(\varepsilon^{-1} x, u^{0}\right)-g\left(\varepsilon^{-1} x, u^{\delta}\right)\right|^{p^{\prime}} d x \leqslant C \delta \quad \forall \varepsilon>0,
$$

где константа $C$ не зависит от $\varepsilon$. Отсюда по лемме 3.1 получаем

$$
\begin{aligned}
\lim _{\delta \rightarrow 0} \lim _{\varepsilon \rightarrow 0} I_{1}^{\varepsilon}= & \lim _{\delta \rightarrow 0} \lim _{\varepsilon \rightarrow 0}\left(\int_{\Omega} g\left(\varepsilon^{-1} x, u^{\delta}\right)\left(u^{\varepsilon}-u^{0}\right) d x\right. \\
& \left.+\int_{\Omega}\left(g\left(\varepsilon^{-1} x, u^{0}\right)-g\left(\varepsilon^{-1} x, u^{\delta}\right)\right)\left(u^{\varepsilon}-u^{0}\right) d x\right)=0 .
\end{aligned}
$$

Таким образом, соотношение (3.23) имеет место.

Поэтому из (3.22) заключаем, что

$$
\lim _{\varepsilon \rightarrow 0} \int_{\Omega} \chi_{\varepsilon}\left(b\left(\varepsilon^{-1} x, u^{\varepsilon}\right)-b\left(\varepsilon^{-1} x, u^{0}\right)\right)\left(u^{\varepsilon}-u^{0}\right) d x=0,
$$

и в силу условия $\mathrm{j}$ ) сильной монотонности $b(x, s)$ отсюда следует утверждение о сильной сходимости решений в смысле (1.15).

4. Теперь докажем свойство сходимости потоков (1.13). Для этого воспользуемся тем, что сильная сходимость решений (1.15) уже доказана, и перепишем исходную задачу (1.1) так:

$$
-\operatorname{div}\left(\chi_{\varepsilon} a\left(\varepsilon^{-1} x, \nabla u^{\varepsilon}\right)\right)=\chi_{\varepsilon} g_{\varepsilon},
$$

где $g_{\varepsilon}=f-b\left(\varepsilon^{-1} x, u^{\varepsilon}\right)$ и выполнены соотношения

$$
\begin{gathered}
\lim _{\varepsilon \rightarrow 0} \int_{\Omega} \chi_{\varepsilon}\left|g_{\varepsilon}-g_{0}\right|^{p^{\prime}} d x=0, \\
\lim _{\varepsilon \rightarrow 0} \int_{\Omega} \chi_{\varepsilon}\left(\left|\nabla u^{\varepsilon}\right|^{p}+\left|u^{\varepsilon}\right|^{p}\right) d x<+\infty, \\
\lim _{\varepsilon \rightarrow 0} \int_{\Omega} \chi_{\varepsilon}\left|u^{\varepsilon}-u^{0}\right|^{p} d x=0,
\end{gathered}
$$


$u^{0}(x)$ - решение усредненной задачи

$$
-\operatorname{div}\left(a_{0}\left(\nabla u^{0}\right)\right)=g_{0}
$$

Пусть $\varphi \in C_{0}^{\infty}(\Omega), \varphi \geqslant 0$. По монотонности имеем

$$
\int_{\Omega} \varphi\left[a\left(\varepsilon^{-1} x, \nabla u^{\varepsilon}\right)-a\left(\varepsilon^{-1} x, \nabla w^{\varepsilon}\right)\right] \cdot\left[\nabla u^{\varepsilon}-\nabla w^{\varepsilon}\right] d x \geqslant 0,
$$

где $w^{\varepsilon}(x)$ - пробная функция, определенная в п. 2.

Это выражение содержит четыре слагаемых. Предел трех из них вычисляется, как и ранее. Теперь мы имеем возможность вычислить предел слагаемого

$$
\mathscr{I}_{\varepsilon}=\int_{\Omega} \chi_{\varepsilon} \varphi a\left(\varepsilon^{-1} x, \nabla u^{\varepsilon}\right) \cdot \nabla u^{\varepsilon} d x
$$

Действительно, из уравнения (3.24) имеем

$$
\int_{\Omega} \varphi \chi_{\varepsilon} a\left(\varepsilon^{-1} x, \nabla u^{\varepsilon}\right) \cdot \nabla u^{\varepsilon} d x+\int_{\Omega} \chi_{\varepsilon} a\left(\varepsilon^{-1} x, \nabla u^{\varepsilon}\right) u^{\varepsilon} \cdot \nabla \varphi d x=\int_{\Omega} \chi_{\varepsilon} g_{\varepsilon} u^{\varepsilon} \varphi d x .
$$

В силу соотношений сильной сходимости (3.25), (3.26)

$$
\lim _{\varepsilon \rightarrow 0} \mathscr{I}_{\varepsilon}=\int_{\Omega} g_{0} u^{0} \varphi d x-\int_{\Omega} p_{0} u^{0} \cdot \nabla \varphi d x
$$

С другой стороны, полагая в интегральном тождестве

$$
\int_{\Omega} \chi_{\varepsilon} a\left(\varepsilon^{-1} x, \nabla u^{\varepsilon}\right) \cdot \nabla \psi=\int_{\Omega} \chi_{\varepsilon} g_{\varepsilon} \psi d x
$$

$\psi=\varphi u^{0}$ и переходя к пределу при $\varepsilon \rightarrow 0$, получим

$$
\int_{\Omega} \varphi p_{0} \cdot \nabla u^{0} d x+\int_{\Omega} p_{0} u^{0} \cdot \nabla \varphi d x=\int_{\Omega} g_{0} u^{0} \varphi d x
$$

Отсюда следует, что

$$
\lim _{\varepsilon \rightarrow 0} \mathscr{I}_{\varepsilon}=\int_{\Omega} \varphi p_{0} \cdot \nabla u^{0} d x
$$

Это позволяет перейти к пределу в неравенстве (3.27) и получить

$$
\int_{\Omega} \varphi\left(p_{0}-a_{0}(\xi)\right) \cdot\left(\nabla u^{0}-\xi\right) d x \geqslant 0 .
$$

Отсюда ввиду произвольности функции $\varphi \geqslant 0$

$$
\left(p_{0}-a_{0}(\xi)\right) \cdot\left(\nabla u^{0}-\xi\right) \geqslant 0 \quad \text { для п.в. } x \in \Omega, \quad \forall \xi \in \mathbb{R}^{N},
$$

и лемма Минти дает искомое равенство $p_{0}=a_{0}\left(\nabla u^{0}\right)$. Тем самым получена слабая сходимость потоков (1.13), и теорема 1.1 доказана. 
5. Как отмечалось в $\S 1$, условия теоремы 1.1 могут быть ослаблены. В частности, для применения к параболическим уравнениям важен случай, когда

$$
b(x, u)=\lambda u(x), \quad \text { где } \lambda>0 .
$$

До сих пор свойства монотонности и свойства роста функций $a(\cdot, \xi), b(\cdot, s)$ характеризовались одним показателем $p$. Теперь, сохраняя показатель $p$ для $a(y, \xi)$, будем характеризовать свойства функции $b(x, s)$ показателем $q>1$, никак не связанньм с $p$, именно для п.в. $x \in Q$ и $\forall s_{1}, s_{2} \in \mathbb{R}^{1} \exists \beta>0$ :

$$
\begin{gathered}
\left(b\left(x, s_{1}\right)-b\left(x, s_{2}\right)\right) \cdot\left(s_{1}-s_{2}\right) \\
\geqslant \begin{cases}\beta\left|s_{1}-s_{2}\right|^{q}, & \text { если } q \geqslant 2, \\
\beta\left|s_{1}-s_{2}\right|^{2}\left(\left|s_{1}\right|+\left|s_{2}\right|\right)^{q-2}, & \text { если } 1<q \leqslant 2,\end{cases} \\
\left|b\left(x, s_{1}\right)-b\left(x, s_{2}\right)\right| \leqslant \begin{cases}\beta^{-1}\left(1+\left|s_{1}\right|+\left|s_{2}\right|\right)^{q-2}\left|s_{1}-s_{2}\right|, & \text { если } q \geqslant 2, \\
\beta^{-1}\left|s_{1}-s_{2}\right|^{q-1}, & \text { если } 1<q \leqslant 2 .\end{cases}
\end{gathered}
$$

Теорема 3.2. Пусть в условиях теоремы 1.1 функиия $b(x, s)$ удовлетворяет условиям (3.29), (3.30). Тогда имеет место слабая сходимость потоков

$$
\begin{aligned}
& \chi_{\varepsilon}(x) a\left(\varepsilon^{-1} x, \nabla u^{\varepsilon}\right) \rightarrow a_{0}\left(\nabla u^{0}\right) \quad \text { слабо в } \quad L^{p^{\prime}}(\Omega), \\
& \chi_{\varepsilon}(x) b\left(\varepsilon^{-1} x, u^{\varepsilon}\right) \rightarrow b_{0}\left(u^{0}\right) \quad \text { слабо в } \quad L^{q^{\prime}}(\Omega),
\end{aligned}
$$

а также справедлива сильная сходимость решений в смысле

$$
\lim _{\varepsilon \rightarrow 0} \int_{\Omega \cap Q_{\varepsilon}}\left|u^{\varepsilon}-u^{0}\right|^{q} d x=0,
$$

где $u^{\varepsilon}$ - решение задачи (1.1), а и $u^{0}$ - решение усредненной задачи (1.12).

ДоКАЗАТЕЛЬСтво. Отметим, что в целом доказательство теоремы 1.1 остается прежним. Изменения касаются только леммы 2.3 и леммы 3.1 .

В лемме 2.3 условия (2.1), (2.3) могут быть ослаблены. В частности, справедлива следуюшая лемма.

ЛЕмма 3.3. Предположим, ито последовательность $u^{\varepsilon} \in C_{0}^{\infty}(\Omega)$ такова, чmo:

1) $\chi_{\varepsilon} u^{\varepsilon}$ слабо компактна в $L^{1}(\Omega)$;

2) $\lim _{\varepsilon \rightarrow 0} \int_{\Omega} \chi_{\varepsilon}\left|\nabla u^{\varepsilon}\right|^{p} d x<+\infty$.

Тогда $u^{0} \in W_{0}^{1, p}(\Omega)$, и если

$$
z \in\left(L_{\mathrm{per}}^{p^{\prime}}(\square)\right)^{N}, \quad \operatorname{div} z=0,\left.\quad z\right|_{\mathbb{R}^{N} \backslash Q}=0,
$$

$m o$ прu $\varepsilon \rightarrow 0$

$$
\nabla u^{\varepsilon} \cdot z\left(\varepsilon^{-1} x\right) \rightarrow \nabla u^{0}\langle z\rangle \quad \text { cлабо в } \quad L^{1}(\Omega) .
$$


ДокАЗАТЕЛЬСТво. Введем срезки

$$
u^{\varepsilon, N}=\left\{\begin{array}{l}
u^{\varepsilon}, \text { если }\left|u^{\varepsilon}\right| \leqslant N, \\
\pm N, \text { если }\left|u^{\varepsilon}\right| \geqslant N,
\end{array}\right.
$$

и положим

$$
K^{\varepsilon, N}=\left\{x \in \Omega \cap Q_{\varepsilon}:\left|u^{\varepsilon}\right| \geqslant N\right\} .
$$

Тогда в силу критерия слабой компактности в $L^{1}(\Omega)$ имеем

$$
\begin{aligned}
\left|K^{\varepsilon, N}\right| & \leqslant \sigma(N), \\
\int_{\Omega} \chi_{\varepsilon}\left|u^{\varepsilon, N}-u^{\varepsilon}\right| d x & \leqslant \sigma(N),
\end{aligned}
$$

где $\lim _{N \rightarrow+\infty} \sigma(N)=0$. Без потери обшности считаем, что при фиксированном $N$ имеет место слабая сходимость

$$
\chi_{\varepsilon} u^{\varepsilon, N} \rightarrow \theta u^{0, N} \quad \text { слабо в } L^{2}(\Omega) \text { при } \varepsilon \rightarrow 0
$$

(в данном случае $u^{0, N}$ - обозначение для слабого предела, а не срезки $u^{0}$ ). Тогда из (3.37) получаем

$$
\lim _{N \rightarrow+\infty} \int_{\Omega}\left|u^{0, N}-u^{0}\right| d x \leqslant \lim _{N \rightarrow+\infty} \sigma(N)=0 .
$$

По лемме 2.3

$$
u^{0, N} \in W_{0}^{1, p}(\Omega), \quad \int_{\Omega}\left|\nabla u^{0, N}\right|^{p} d x \leqslant c_{0},
$$

где $c_{0}$ не зависит от $N$. Поэтому последовательность $u^{0, N}$ ограничена в $W_{0}^{1, p}(\Omega)$ при $N \rightarrow+\infty$. Согласно (3.38)

$$
u^{0, N} \rightarrow u^{0} \quad \text { слабо в } W_{0}^{1, p}(\Omega)
$$

и, следовательно, $u^{0} \in W_{0}^{1, p}(\Omega)$.

Теперь докажем свойство (3.34). Легко проверить (аналогично доказательству леммы 2.2), что семейство $\nabla u^{\varepsilon} \cdot z_{\varepsilon}$, где $z_{\varepsilon}=z\left(\varepsilon^{-1} x\right)$, слабо компактно в $L^{1}(\Omega)$. Поэтому для доказательства (3.34) достаточно доказать соотношение

$$
\lim _{\varepsilon \rightarrow 0} \int_{\Omega} \varphi \nabla u^{\varepsilon} \cdot z_{\varepsilon} d x=\int_{\Omega} \varphi \nabla u^{0} \cdot\langle z\rangle d x \quad \forall \varphi \in C_{0}^{\infty}(\Omega) .
$$

Имеем

$$
\begin{aligned}
\left|\mathscr{I}^{\varepsilon}-\mathscr{I}^{\varepsilon, N}\right| \stackrel{\text { def }}{=}\left|\int_{\Omega \cap Q_{\varepsilon}} \varphi \nabla u^{\varepsilon} \cdot z_{\varepsilon} d x-\int_{\Omega \cap Q_{\varepsilon}} \varphi \nabla u^{\varepsilon, N} \cdot z_{\varepsilon} d x\right| \\
=\left|\int_{K^{\varepsilon, N}} \varphi \nabla u^{\varepsilon} \cdot z_{\varepsilon} d x\right| \leqslant \sup |\varphi|\left(\int_{\Omega \cap Q_{\varepsilon}}\left|\nabla u^{\varepsilon}\right|^{p} d x\right)^{1 / p} \\
\quad \times\left(\int_{K^{\varepsilon, N}}\left|z_{\varepsilon}\right|^{p^{\prime}} d x\right)^{1 / p^{\prime}} \leqslant C\left(\int_{K^{\varepsilon, N}}\left|z_{\varepsilon}\right|^{p^{\prime}} d x\right)^{1 / p^{\prime}} \leqslant \gamma(N) .
\end{aligned}
$$


Так как семейство функций $\left|z_{\varepsilon}\right|^{p^{\prime}}$ равностепенно интегрируемо, то $\lim _{N \rightarrow+\infty} \gamma(N)=0$. При фиксированном $N>0$ функция $u^{\varepsilon, N}$, очевидно, удовлетворяет условиям леммы 2.3. Поэтому

$$
\lim _{\varepsilon \rightarrow 0} \mathscr{I}^{\varepsilon, N}=\lim _{\varepsilon \rightarrow 0} \int_{\Omega \cap Q_{\varepsilon}} \varphi \nabla u^{\varepsilon, N} \cdot z_{\varepsilon} d x=\int_{\Omega} \varphi \nabla u^{0, N} \cdot\langle z\rangle d x \quad \forall \varphi \in L^{\infty}(\Omega) .
$$

Остается в последнем равенстве перейти к пределу при $N \rightarrow+\infty$, используя сходимость (3.39). Лемма доказана.

Теперь дадим подходящее обобщение леммы 3.1 .

ЛЕмма 3.4. Пусть $d(x) \in L_{\text {per }}^{q^{\prime}}(\square), \quad q^{\prime}=\frac{q}{q-1},\left.\quad d\right|_{\mathbb{R}^{N} \backslash Q}=0, \quad\langle d\rangle=0 . \quad$ Ecлu $w^{\varepsilon} \in C_{0}^{\infty}(\Omega) u$

$$
\lim _{\varepsilon \rightarrow 0} \int_{\Omega} \chi_{\varepsilon}\left(\left|w^{\varepsilon}\right|^{q}+\left|\nabla w^{\varepsilon}\right|\right) d x<+\infty
$$

mo

$$
\lim _{\varepsilon \rightarrow 0} \int_{\Omega} \chi_{\varepsilon} d\left(\varepsilon^{-1} x\right) w^{\varepsilon}(x) d x=0
$$

ДокАЗАТЕльство. В [6, с. 405] показано, что справедливо следуюшее представление:

$$
\forall \delta>0 \quad d(x)=d_{0}(x)+\frac{\partial}{\partial x_{i}} d_{i}(x) \quad \text { в } \quad \mathbb{R}^{N}
$$

где

$$
d_{i} \in C_{\mathrm{per}}^{\infty}(\square),\left.\quad d_{i}\right|_{\square \backslash Q}=0, \quad i=1, \ldots, N, \quad\left\|d_{0}\right\|_{L^{q^{\prime}(\Omega)}}^{q^{\prime}} \leqslant \delta
$$

Поэтому, используя (3.40), получим

$$
\int_{\Omega} \chi_{\varepsilon} d\left(\varepsilon^{-1} x\right) w^{\varepsilon}(x) d x=\int_{\Omega \cap Q_{\varepsilon}} d_{0}\left(\varepsilon^{-1} x\right) w^{\varepsilon}(x) d x-\varepsilon \int_{\Omega \cap Q_{\varepsilon}} d_{i}\left(\varepsilon^{-1} x\right) \frac{\partial w^{\varepsilon}}{\partial x_{i}} d x
$$

Рассмотрим первое слагаемое в (3.41). Имеем

$$
\begin{aligned}
& \lim _{\varepsilon \rightarrow 0}\left|\int_{\Omega} d_{0}\left(\varepsilon^{-1} x\right) w^{\varepsilon}(x) d x\right| \leqslant \lim _{\varepsilon \rightarrow 0}\left(\int_{\Omega \cap Q_{\varepsilon}}\left|d_{0}\left(\varepsilon^{-1} x\right)\right|^{q^{\prime}} d x\right)^{1 / q^{\prime}} \\
& \times\left(\int_{\Omega} \chi_{\varepsilon}\left|w^{\varepsilon}\right|^{q} d x\right)^{1 / q} \leqslant C|\Omega|\left(\int_{\square \cap Q}\left|d_{0}\right|^{q^{\prime}} d x\right)^{1 / q^{\prime}} \leqslant C|\Omega| \delta
\end{aligned}
$$

по свойству среднего значения.

Аналогично устанавливается, что второе слагаемое в (3.41) стремится к нулю при $\varepsilon \rightarrow 0$. Лемма 3.4 доказана.

В остальном доказательство теоремы 1.1 остается без изменений. 


\section{§4. Свойства усредненного оператора}

Докажем некоторые свойства усредненного оператора $a_{0}(\xi)$, указанные в $\S 1$.

1. Во-первых, очевидно, что ввиду сильной монотонности $a(y, \xi)$ из определения $a_{0}$ (см. (1.9)) и свойств і) следует, что усредненный оператор будет строго монотонныт.

Во-вторых, покажем, что справедлива оценка

$$
a_{0}(\xi) \cdot \xi \geqslant \alpha_{0}|\xi|^{p}, \quad \alpha_{0}>0,
$$

обеспечивающая коэрцитивность $a_{0}$.

Действительно, из (1.9), учитьвая условие 3$)$ и свойства і) для $a(y, \xi)$, имеем

$$
a_{0}(\xi) \cdot \xi=\int_{\square} \chi(y)(a(y, \xi+v)-a(y, 0)) \cdot \xi d y \geqslant \alpha \int_{\square} \chi|\xi+v|^{p} d y .
$$

Но для вариационной задачи

$$
f_{0}(\xi)=\min \int_{\square} \chi(y)|\xi+v(y)|^{p} d y
$$

известно, что $f_{0}$ коэрцитивен (см. [6]), т.е.

$$
f_{0}(\xi) \geqslant c_{0}|\xi|^{p}, \quad c_{0}>0 \quad\left(\forall \xi \in \mathbb{R}^{N}\right),
$$

поэтому отсюда и из (4.2) сразу получаем неравенство (4.1).

Таким образом, коэрцитивность усредненного оператора $a_{0}$ доказана.

2. Получим теперь оценки типа іi) для усредненного оператора. Предварительно докажем следующую лемму.

ЛЕмма 4.1. Имеем

$$
\left|a_{0}(\xi)\right| \leqslant c(1+|\xi|)^{p-1},
$$

әде $c=c(\alpha, p)$.

ДокАЗАТЕЛЬСТВО. Пусть $v(y, \xi)$ - решение вспомогательной периодической задачи (1.7) для фиксированного $\xi$. Тогда в силу іi) имеем

$$
\left|a_{0}(\xi)-\int_{\square} a(y, 0) d y\right| \leqslant \alpha^{-1} \int_{\square}(1+|z|)^{p-1} d y,
$$

где мы положили $z(y, \xi)=\xi+v(y, \xi)$.

С другой стороны, учитьвая сильную монотонность і) и условие 3 ), получим

$$
\alpha \int_{\square}|z|^{p} d y \leqslant \int_{\square}(a(y, z)-a(y, 0)) \cdot z d y=a_{0}(\xi) \cdot \xi .
$$

Отсюда и из (4.4) следует, что

$$
\int_{\square}|z|^{p} d y \leqslant c(1+|\xi|)^{p}, \quad c=c(\alpha, p) .
$$

Снова возврашаясь к (4.4), заключаем, что неравенство (4.3) имеет место. Лемма 4.1 доказана.

Пусть $v_{1}=v\left(y, \xi_{1}\right)$ и $v_{2}=v\left(y, \xi_{2}\right)$ - решения вспомогательной периодической задачи (1.7) для $\xi_{1}$ и $\xi_{2}$ соответственно. Положим также $z_{1}=\xi_{1}+v_{1}, z_{2}=\xi_{2}+v_{2}$. Тогда справедлива следуюшая 
Лемма 4.2. Имеем $\forall \xi_{1}, \xi_{2} \in \mathbb{R}^{N}$

$$
\left|a_{0}\left(\xi_{1}\right)-a_{0}\left(\xi_{2}\right)\right| \leqslant\left\{\begin{array}{lll}
c\left(1+\left|\xi_{1}\right|+\left|\xi_{2}\right|\right)^{\frac{p(p-2)}{p-1}}\left|\xi_{1}-\xi_{2}\right|^{\frac{1}{p-1}}, & \text { если } & p \geqslant 2, \\
c\left(1+\left|\xi_{1}\right|+\left|\xi_{2}\right|\right)^{\frac{(2-p)(p-1)}{3-p}}\left|\xi_{1}-\xi_{2}\right|^{\frac{p-2}{3-p}}, & \text { еслu } & 1<p \leqslant 2,
\end{array}\right.
$$

əде $c=c(\alpha, p)$.

ДокАЗАТЕльство. Рассмотрим случай $p \geqslant 2$. Используя сильную монотонность i) функции $a(y, \xi)$ и применяя неравенство Гёльдера, получим

$$
\begin{aligned}
\alpha \int_{\square}\left|z_{1}-z_{2}\right|^{p} d y & \leqslant \int_{\square}\left(a\left(y, z_{1}\right)-a\left(y, z_{2}\right)\right) \cdot\left(z_{1}-z_{2}\right) d y \\
& \leqslant\left|\xi_{1}-\xi_{2}\right|^{\frac{p}{p-1}}\left(1+\left|\xi_{1}\right|+\left|\xi_{2}\right|\right)^{\frac{p(p-2)}{p-1}} .
\end{aligned}
$$

С другой стороны, согласно іi) и (4.5) имеем

$$
\begin{aligned}
\left|a_{0}\left(\xi_{1}\right)-a_{0}\left(\xi_{2}\right)\right| & =\int_{\square}\left|a\left(y, z_{1}\right)-a\left(y, z_{2}\right)\right| d y \\
& \leqslant c\left(1+\left|\xi_{1}\right|+\left|\xi_{2}\right|\right)^{p-2}\left(\int_{\square}\left|z_{1}-z_{2}\right|^{p} d y\right)^{1 / p} .
\end{aligned}
$$

Отсюда и из оценки (4.7) получаем первое неравенство (4.6).

Рассуждая аналогично в случае $1<p \leqslant 2$ и учитывая соответствуюшее условие і), имеем

$$
\alpha \int_{\square}\left|z_{1}-z_{2}\right|^{2}\left(\left|z_{1}\right|+\left|z_{2}\right|\right)^{p-2} d y \leqslant \int_{\square}\left(a\left(y, z_{1}\right)-a\left(y, z_{2}\right)\right) \cdot\left(z_{1}-z_{2}\right) d y .
$$

Далее применим к левой части (4.8) обратное неравенство Гёльдера и воспользуемся оценкой

$$
\left|a_{0}\left(\xi_{1}\right)-a_{0}\left(\xi_{2}\right)\right| \leqslant \alpha^{-1} \int_{\square}\left|z_{1}-z_{2}\right|^{p-1} d y
$$

которая следует из соответствуюшего условия ii) для $a(y, \xi)$. Тогда из $(4.8),(4.9)$, принимая во внимание оценку (4.5), заключаем, что и второе неравенство (4.6) имеет место. Лемма 4.2 доказана. 


\section{Список литературы}

1. Лионс Ж. Л. Некоторые методы решения нелинейных краевых задач. М.: Мир, 1972.

2. Дубинский Ю. А. Квазилинейные эллиптические и параболические уравнения любого порядка // УМН. 1968. Т. 23. №1. С. 45-90.

3. Панков $A . A$. Об усреднении и $G$-сходимости нелинейных эллиптических операторов // ДАН СССР. 1984. Т. 278. № 1. С. 37-41.

4. Fusko N., Moskariello G. On the homogenization of quasilinear divergence structure operators // Ann. Mat. Pura Appl. 1987. V. 147. P. 1-13.

5. Tartar L. Cours Peccot au College de France. Paris, 1977.

6. Zhikov $V$. V. On the homogenization of nonlinear variational problems in perforated domains // Russian J. of mathem. physics. 1994. V. 2. № 3. P. 393-408.

7. Жиков В.В. Об усреднении в перфорированных случайных областях общего вида // Матем. заметки. 1993. Т. 53. №1. С. 41-58.

8. Жиков В.В. Усреднение функционалов вариационного исчисления и теории упругости // Изв. АН СССР. Сер. матем. 1986. Т. 50. № 4. С. 675-711.

9. Жиков В.В., Козлов С.М., Олейник О.А. Усреднение дифференциальных операторов. М.: Наука, 1993.

10. Олейник O.А., Иосифьян Г. А., Шамаев А. С. Математические задачи теории сильно неоднородных упругих сред. М.: Изд-во МГУ, 1990.

11. Acerbi E., Chiado Piat V., Dal Maso G., Percivale D. An extension theorem for connected sets and homogenization in general periodic domains // Nonlinear Anal. 1992. V. 18. № 5. P. 481-496.

12. Chiado Piat V., Defranceschi A. Homogenization of monotone operators // Nonlinear Anal. 1990. V. 14. P. 717-732.

13. Экланд И., Темам Р. Выпуклый анализ и вариационные проблемы. М.: Мир, 1979.

Поступило в редакцию 10.VII. 1995 